\title{
Possible Reason for the High Metabolic Rate in Neurons of a Brain
}

J ingjing $\mathrm{Xu}^{1}$ and Shengyong $\mathrm{Xu}^{2 *}$

${ }^{1}$ Institute of Microelectronics, Shandong University, China

${ }^{2}$ Key Laboratory for the Physics \& Chemistry of

Nanodevices, and Department of Electronics, Peking

University, China

*Corresponding author: Shengyong Xu, Key

Laboratory for the Physics \& Chemistry of Nanodevices, and Department of Electronics, School of Electronics

Engineering and Computer Science, Peking University, China

Received: December 10, 2018; Accepted: December 26, 2018; Published: December 31, 2018

\section{Editorial}

It has been found that the brain of an adult consumes about $20 \%$ of the total daily energy consumption of his whole body, although the brain cells weighs only a few presents of the body. In some highly intensive brain activities, such as in chess or go games, math competition, SAT examinations, it is estimated that the brain may consume more than $50 \%$ of the total energy consumption. What is the reason? And how does it work that the average metabolic rate of brain cells may be 5-10 times higher than that of other cells of the body?

We believe that the answer lies in the working function of brain cells, in particular, the neurons. Neuron cells in a brain works in the similar way to that of the neurons in the peripheral neural systems, e.g., the spine. For a certain moment, a neuron cell is either in an excitation state, or in a resting state. We may assume that a neuron in resting state has the same metabolic rate as those of other body cells. While in its excitation state, a neuron cell consumes more energy than in its resting state. Experiments showed that a single excitation of a neurosome, the main cell body, only lasts for 1-2 ms. Yet when playing a chess game or giving a speech, one needs seconds, minutes and even hours to analyze the dynamically acquired new information, perform comparisons with existing memory, and make decisions. These brain activities require that short memories have to maintains right information data for seconds to hours. One sees here a huge mismatch in time scale: Between a few milliseconds and minutes or hours, a gap of 3-7 orders of magnitude. The nature should have found a way to solve the mismatch in time. Then, what is the feasible working mechanism?

We have presented a hypothesis, that the information restored in a brain are memorized in the forms of "two-dimensional (2D) patterns" of neurosomes, where each two neighboring neurosomes are strongly connected with their synapses [1]. According to this model, after receiving information from various body sensors, in the Sensory Layer (S) of the cortex certain groups of neurosomes are excited, forming a 2D pattern, as schematically illustrated in (Figure

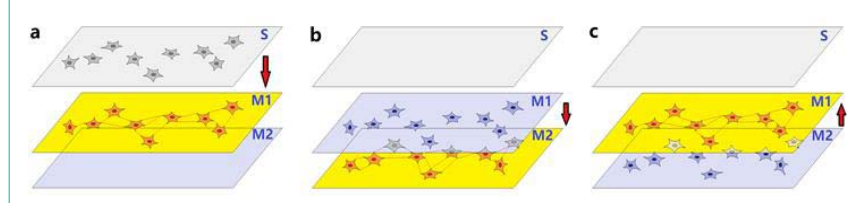

Figure 1: Schematic illustration of the forming and echoing processes for temporary memory. a) A 2D pattern of excited neurosomes (highlighted with grey stars) is formed on the Sensory Layer (S), corresponding to the data obtained from body sensors. This neurosome pattern projected into the first Memory Layer (M1) through dendrites, causing simultaneous excitation of neurons in this layer (orange stars) with a pattern similar to that on S layer. b) The $2 \mathrm{D}$ pattern is further projected to the second Memory Layer (M2) through dendrites. c) To maintain the information, the 2D pattern of excited neurosomes is echoed back to M1 layer. Repeating b) and c) keeps the data stored in the 2D pattern as temporary memory.

1a). This $2 \mathrm{D}$ pattern in the sensory layer then projects into a Memory Layer (M1) through the vertical dendrites, as shown in (Figure 1b). The pattern is copied based on the fact that the synaptic connection between two neurosomes is strengthened when it receives electrical signals from the two neurons simultaneously. The sensory layer is cleared for receiving next sensing data. Then the 2D pattern copied in $\mathrm{M} 1$ is further projected to a second Memory Layer (M2), as shown in (Figure 1c). To maintain the data stored in the 2D pattern, the pattern should be echoed back to M1, and the processes of $1 \mathrm{~b}$ and $1 \mathrm{c}$ need to repeat again and again. This echoing and oscillating mechanism between memory layers $(\mathrm{M} 1 \rightarrow \mathrm{M} 2 \rightarrow \mathrm{M} 1 \rightarrow \mathrm{M} 2 \rightarrow \ldots \ldots)$ ensures a temporary memory at the seconds and minutes level, in spite of the short transient excitation duration for each layer of neurons (i.e., a few ms).

Between M1 and M2 layers, one echoing process through dendrites in a local area of cortex presumably takes less than 10 ms. But in complicated brain functions, echoing processes could take place among different regions of the cortex, across a space up to $10 \mathrm{~cm}$ or more. Assuming the transmission speed in brain axons is around $10 \mathrm{~m} / \mathrm{s}$, it may take $10 \mathrm{~ms}$ for a one-way propagation and 20-30 ms for a complete echoing process. Therefore, for neurons involved in memory processes could be estimated to excite 30-100 times per second. For millions and billions of neurons working at the same time in intensive brain functions, it will lead to averaging frequencies of electrical neural signals. And this is consistent with the EEG experimental data.

Our model suggests that in the tens of billions of neurons in an adult brain, only a small portion is responsible for functions of comparison, analysis, making decision etc. [2]. Most neurons are probably involved in storage of temporary and long-term memory information. For both storing and retrieving the memory information, it needs echoing processes between two layers of neurosomes. For maintaining the echoing processes, the neurons involve in the $2 \mathrm{D}$ patterns need to continuously consume energy. The energy is mainly 
cost for continuous activating of ionic pumps (eg., $\mathrm{Na}^{+}-\mathrm{K}^{+}$pumps) embedded in the membrane so as to reset the transmembrane gradients in concentration of various ions. As a result, these neurons show a much higher metabolic rate than that of normal cells.

To verify the hypothesis, one need to observe in vivo the oscillation phenomenon between M1 and M2, which is feasible but quite a technical challenge.

\section{Acknowledgement}

This work is financially supported by National Key R\&D Program of China No. 2017YFA0701302.

\section{References}

1. Shengyong $\mathrm{Xu}$ and Jingjing $\mathrm{Xu}$, "A memory mechanism based on two dimensional code of neurosome pattern"; Proceedings for International Conference on Biological Information and Biomedical Engineering (BIBE 2018), VDE Verlag GmbH.

2. Shengyong $\mathrm{Xu}$, Jingjing $\mathrm{Xu}$ and Rujun Dai, "Layered structure and leveled function of a human brain".
Austin J Womens Health - Volume 5 Issue 1 - 2018

Submit your Manuscript | www.austinpublishinggroup.com

$\mathrm{Xu}$ et al. (C) All rights are reserved
Citation: Xu J and Xu S. Possible Reason for the High Metabolic Rate in Neurons of a Brain. Austin J Womens Health. 2018; 5(1): 1031. 